\title{
Comparing the cost-effectiveness of FOLFIRINOX, nab-paclitaxel plus gemcitabine, gemcitabine and $S-1$ for the treatment of metastatic pancreatic cancer
}

\author{
MACHIKO KURIMOTO, MICHIO KIMURA, EISEKI USAMI, MINA IWAI, \\ TATSUYA HIROSE, SHIORI KAWACHI and TOMOAKI YOSHIMURA \\ Department of Pharmacy, Ogaki Municipal Hospital, Ogaki, Gifu 503-8502, Japan
}

Received January 4, 2017; Accepted March 22, 2017

DOI: $10.3892 / \mathrm{mco} .2017 .1278$

\begin{abstract}
The recommended chemotherapy regimens for pancreatic cancer include the combination of 5-fluorouracil/ leucovorin, oxaliplatin and irinotecan (FOLFIRINOX), nab-paclitaxel (nab-PTX) plus gemcitabine (GEM), GEM alone and tegafur/gimeracil/oteracil potassium (S-1) alone. Although the cost-effectiveness of metastatic pancreatic cancer chemotherapies has been extensively investigated, to the best of our knowledge, no study has specifically compared the cost-effectiveness among FOLFIRINOX, nab-PTX + GEM, GEM and S-1 regimens to date. The aim of the present study was to examine the cost-effectiveness of these four regimens. The expected costs were calculated based on data from patients with metastatic pancreatic cancer who were treated with the FOLFIRINOX, nab-PTX + GEM, GEM alone or S-1 alone. The median survival time (MST) from randomized controlled trials in the literature was used to evaluate the therapeutic effect of these regimens. The cost-effectiveness ratio was calculated using expected costs and MST for these four regimens. The expected costs per patient for the FOLFIRINOX, nab-PTX + GEM, GEM or S-1 regimens were $¥ 6,361,191.4, ¥ 4,802,063.6, ¥ 540,091.4$ and $¥ 528,514.6$, respectively, and the cost-effectiveness ratios per month were $¥ 642,544.6 / \mathrm{MST}, ¥ 470,790.5 / \mathrm{MST}$, $¥ 81,832.0 / \mathrm{MST}$ and $¥ 55,633.1 / \mathrm{MST}$, respectively. In conclusion, the nab-PTX + GEM and FOLFIRINOX regimens were associated with a high therapeutic efficacy and high cost. The GEM regimen exhibited a lower therapeutic efficacy compared with the nab-PTX + GEM and FOLFIRINOX regimens, but the findings of this study indicated that the GEM and $\mathrm{S}-1$ regimens were the most cost-effective regimens.
\end{abstract}

\footnotetext{
Correspondence to: Dr Machiko Kurimoto, Department of Pharmacy, Ogaki Municipal Hospital, 4-86 Minaminokawa-cho, Ogaki, Gifu 503-8502, Japan

E-mail: johnnosuke1029@yahoo.co.jp
}

Key words: FOLFIRINOX, nab-paclitaxel, gemcitabine, S-1, cost-effectiveness, adverse event, metastatic pancreatic cancer

\section{Introduction}

The recommended chemotherapy regimens for pancreatic cancer include the combination of 5-fluorouracil (5-FU)/leucovorin (FU/LV), oxaliplatin and irinotecan (FOLFIRINOX), nab-paclitaxel (nab-PTX) plus gemcitabine (GEM), GEM alone and tegafur/gimeracil/oteracil potassium (S-1) alone. The FOLFIRINOX and nab-PTX+GEM regimens have shown a particularly high effectiveness in the treatment of pancreatic cancer with distant metastases (1-4). According to the guidelines of the Japan Society of Clinical Oncology, the FOLFIRINOX and nab-PTX+GEM regimens are considered to be the first choice of treatment for metastatic pancreatic cancer (5). However, when these first-choice regimens are not viable, an appropriate treatment is selected among the GEM, erlotinib plus GEM, or S-1 regimens.

Chemotherapy regimens for the treatment of colon cancer, such as FU/LV + oxaliplatin/capecitabine + oxaliplatin $($ FOLFOX/CapeOX) \pm bevacizumab, the combination of FU/LV and irinotecan (FOLFIRI) \pm cetuximab, and FOLFOX \pm cetuximab, have been reported to prolong survival (4-7). However, the high medical cost of these treatments has often been discussed (8). There are similar concerns regarding the cost of future pancreatic cancer chemotherapies. Although the cost-effectiveness of metastatic pancreatic cancer chemotherapies has been previously investigated (9-13), to the best of our knowledge, no study has specifically compared the cost-effectiveness among FOLFIRINOX, nab-PTX + GEM, GEM and S-1 regimens to date.

The aim of the present study was to evaluate the cost-effectiveness of FOLFIRINOX, nab-PTX + GEM, GEM and S-1 regimens as treatments for metastatic pancreatic cancer.

\section{Patients and methods}

Treatment regimen. The FOLFIRINOX regimen was administered as follows: Oxaliplatin $\left(85 \mathrm{mg} / \mathrm{m}^{2}\right.$ over $\left.2 \mathrm{~h}\right)$, leucovorin (200 mg/m $\mathrm{m}^{2}$ over $\left.2 \mathrm{~h}\right)$, irinotecan $\left(180 \mathrm{mg} / \mathrm{m}^{2}\right.$ over $\left.90 \mathrm{~min}\right)$ and 5 -FU $\left(400 \mathrm{mg} / \mathrm{m}^{2}\right.$ bolus followed by $2,400 \mathrm{mg} / \mathrm{m}^{2}$ over $46 \mathrm{~h})$, all on day 1 , and then repeated every 2 weeks. The nab-PTX + GEM regimen comprised nab-PTX $\left(125 \mathrm{mg} / \mathrm{m}^{2}\right)$ followed by GEM $\left(1,000 \mathrm{mg} / \mathrm{m}^{2}\right)$ administered on days 1,8 and 
Table I. Patient characteristics.

\begin{tabular}{|c|c|c|c|c|c|}
\hline \multirow[b]{2}{*}{ Characteristics } & \multicolumn{5}{|c|}{ Regimens } \\
\hline & $\begin{array}{l}\text { FOLFIRINOX } \\
\qquad(\mathrm{n}=6)\end{array}$ & $\begin{array}{c}\text { nab-PTX + GEM } \\
(\mathrm{n}=18)\end{array}$ & GEM $(n=11)$ & S-1 (n=9) & P-value \\
\hline Age, years & & & & & 0.060 \\
\hline Median (range) & $65.0(62-69)$ & $65.5(44-77)$ & $73.2(52-82)$ & $68.8(55-88)$ & \\
\hline Gender & & & & & 0.519 \\
\hline Male & 4 & 11 & 5 & 7 & \\
\hline Female & 2 & 7 & 6 & 2 & \\
\hline ECOG performance status & & & & & 0.672 \\
\hline 0 & 4 & 12 & 7 & 5 & \\
\hline 1 & 2 & 6 & 4 & 3 & \\
\hline 2 & 0 & 0 & 0 & 1 & \\
\hline \multicolumn{6}{|l|}{ Body surface area, $\mathrm{m}^{2}$} \\
\hline Median (range) & $1.48(1.25-1.83)$ & $1.57(1.24-1.95)$ & $1.48(1.30-1.63)$ & $1.50(1.29-1.75)$ & 0.441 \\
\hline Creatinine clearance, $\mathrm{ml} / \mathrm{mi}$ & & & & & \\
\hline Median (range) & $87.1(52.1-112.3)$ & $81.4(36.4-139.9)$ & $71.2(48.3-94.9)$ & $85.3(52.3-125.1)$ & 0.566 \\
\hline Relative dose intensity, $\%$ & & & & & \\
\hline Median (range) & $63.2(57.1-80.0)$ & $72.5(36.7-100)$ & $87.6(66.7-100)$ & $78.1(66.7-100)$ & 0.126 \\
\hline Metastatic site & & & & & 0.193 \\
\hline Liver & 4 & 9 & 8 & 1 & \\
\hline Lung & 0 & 4 & 1 & 2 & \\
\hline Peritoneum & 0 & 7 & 3 & 3 & \\
\hline Lymph nodes & 2 & 2 & 1 & 3 & \\
\hline Adrenal gland & 0 & 0 & 0 & 1 & \\
\hline
\end{tabular}

FOLFIRINOX, 5-fluorouracil/leucovorin, oxaliplatin and irinotecan; nab-PTX, nab-paclitaxel; GEM, gemcitabine; S-1, tegafur/gimeracil/oteracil potassium.

15 every 4 weeks. In the GEM regimen, GEM $\left(1,000 \mathrm{mg} / \mathrm{m}^{2}\right.$ intravenously over $30 \mathrm{~min}$ ) was administered on days 1,8 and 15 every 4 weeks. Finally, in the S-1 regimen, S-1 was administered at a dose of 80,100 or $120 \mathrm{mg} /$ day according to the body-surface area on days 1-14 of a 28-day cycle.

Literature review. A literature review was performed to obtain clinical information in order to calculate the probability of the efficacy of each chemotherapy regimen. The search was performed as of September, 2016, using PubMed as a document retrieval system. The search used key words including 'pancreas cancer', 'FOLFIRINOX', 'nab-paclitaxel plus gemcitabine', 'gemcitabine' and 'S-1', and was narrowed down to include randomized controlled trials.

Cost-effectiveness analysis. Patients were administered $\geq 2$ courses of the FOLFIRINOX $(n=6)$, nab-PTX + GEM $(n=18)$, GEM $(n=11)$ or $S-1(n=9)$ regimens for the treatment of metastatic pancreatic cancer.

Cost data. The cost data included direct costs incurred at the time of chemotherapy. Fees for medication (including supportive care), inspection and outpatient medical examination were calculated. Information on drug prices was collected from the Insurance Drug Encyclopedia (14) and on medical fees from the Medical Fee Points Table (15) to calculate total medical expenses.

Calculation exclusions. The diagnostic imaging (chest computed tomography scan) costs and the labor costs of the medical staff are included in each chemotherapy treatment. These costs were excluded from the calculations in this analysis. The running and depreciation costs of facilities were also excluded, as they are difficult to dispense per patient.

Cost-effectiveness. The cost-effectiveness analysis was conducted by examining the cost and effectiveness data of each chemotherapy obtained using the abovementioned methods. The cost-effectiveness ratio of each chemotherapy was calculated by dividing the expected cost by the median survival time (MST).

Adverse event analysis. AEs were retrospectively investigated for each patient. The date for each AE was identified using electronic charts and pharmacy service records. The severity of AEs was classified according to the Common Terminology Criteria for Adverse Events (http://www.acrin. org/Portals/0/Administration/Regulatory/CTCAE_4.02_2009-09-15 _QuickReference_5X7.pdf) (16). 
Table II. Detailed cost data.

\begin{tabular}{lcccc}
\hline & \multicolumn{3}{c}{ Regimens } \\
\cline { 2 - 5 } Variables & FOLFIRINOX & nab-PTX + GEM & GEM & S-1 \\
\hline Medication fee & & & & \\
Anticancer drugs & $¥ 281,329.0$ & $¥ 426,716.8$ & $¥ 63,682.6$ & $¥ 67,353.1$ \\
Supportive care drugs & $¥ 12,137.2$ & $¥ 1,317.8$ & $¥ 364.3$ & $¥ 515.2$ \\
Inspection fee & $¥ 1,170.0$ & $¥ 3,705.0$ & $¥ 4,095.0$ & $¥ 2,730.0$ \\
Outpatient medical examination fee & $¥ 730.0$ & $¥ 2,676.7$ & $¥ 26,462.5$ & $¥ 1,743.9$ \\
Malignant tumourspecific substances, therapeutic & $¥ 5,000.0$ & $¥ 5,000.0$ & $¥ 5,000.0$ & $¥ 4,000.0$ \\
and management fee & & & & \\
Others & $¥ 690.0$ & $¥ 1,150.0$ & $¥ 603.8$ & $¥ 1,495.0$ \\
Total & $¥ 301,056.2$ & $¥ 440,566.3$ & $¥ 100,208.2$ & $¥ 77,837.2$ \\
\hline
\end{tabular}

The cost of each regimen per course is shown. FOLFIRINOX, 5-fluorouracil/leucovorin, oxaliplatin and irinotecan; nab-PTX, nabpaclitaxel; GEM, gemcitabine; S-1, tegafur/gimeracil/oteracil potassium.

Table III. Cost-effectiveness ratio.

\begin{tabular}{|c|c|c|c|c|c|}
\hline \multirow[b]{2}{*}{ Variables } & \multicolumn{5}{|c|}{ Regimens } \\
\hline & FOLFIRINOX & nab-PTX + GEM & GEM & S-1 & P-value \\
\hline Expected cost per patient & $¥ 6,361,191.4$ & $¥ 4,802,063.6$ & $¥ 540,091.4$ & $¥ 528,514.6$ & $<0.001^{\mathrm{b}}$ \\
\hline MST (months) & 9.9 & 10.2 & 6.6 & 9.5 & $<0.001^{\mathrm{d}}$ \\
\hline Cost-effectiveness ratio ${ }^{\mathrm{a}}$ & $¥ 642,544.6$ & $¥ 470,790.5$ & $¥ 81,832.0$ & $¥ 55,633.1$ & $<0.001^{\mathrm{c}}$ \\
\hline \multicolumn{6}{|c|}{ 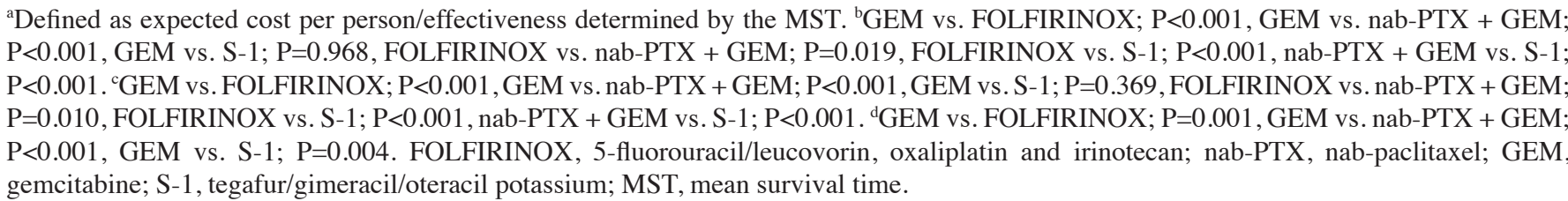 } \\
\hline
\end{tabular}

Statistical analysis. One-factor analysis of variance was used to analyze patient characteristics (Table I). The Fisher Protected Least Significant Difference was used to analyze the variables shown in Table III. In all significance tests, $\mathrm{P}<0.05$ was considered to indicate statistical significance. All statistical analyses were performed using JMP 8 software (SAS Institute Inc, Cary, NC, USA).

Ethical considerations. This study was approved by the Institutional Review Board of Ogaki Municipal Hospital, Ogaki, Japan.

\section{Results}

Patient characteristics. The patient characteristics are summarized in Table I. The median age of the patients who received the FOLFIRINOX, nab-PTX + GEM, GEM and S-1 regimens was 65.0 (range, 62-69), 65.5 (range, 44-77), 73.2 (range, 52-82) and 68.8 (range, 55-88) years, respectively.

Cost data (cost per course of chemotherapy). For the FOLFIRINOX regimen, the calculated direct medical costs included a medication fee (anticancer drugs $=¥ 281,329.0$ and supportive care drugs $=¥ 12,137.2)$, inspection fee $(¥ 1,170.0)$ and outpatient medical examination fee (¥730.0). For the nab-PTX + GEM regimen, the calculated direct medical costs included a medication fee (anticancer drugs $=¥ 426,716.8$ and supportive care drugs $=¥ 1,317.8)$, inspection fee $(¥ 3,705.0)$ and outpatient fee (¥2,676.7). For the GEM regimen, the calculated direct medical costs included a medication fee (anticancer drugs $=¥ 63,682.6$ and supportive care drugs $=¥ 364.3)$, inspection fee $(¥ 4,095.0)$ and outpatient fee $(¥ 26,462.5)$. For the S-1 regimen, the calculated direct medical costs included a medication fee (anticancer drugs $=¥ 67,353.1$ and supportive care drugs $=¥ 515.2)$, inspection fee $(¥ 2,730.0)$ and outpatient fee $(¥ 1,743.9)$.

As regards supportive care drugs, they primarily included prescriptions for antiemetics, laxatives, diuretics and gargle solutions. The prescriptions of aprepitant and pregabalin significantly raised the cost of the supportive care in the FOLFIRINOX regimen.

The nab-PTX + GEM regimen had the highest medication fee among all regimens and the FOLFIRINOX regimen had the highest cost for supportive care drugs (Table II). 
Table IV. Adverse events.

A, FOLFIRINOX $(n=6)$

\begin{tabular}{|c|c|c|c|c|c|}
\hline \multirow[b]{2}{*}{ Toxicities } & \multicolumn{4}{|c|}{ Grade, $\mathrm{n}$} & \multirow{2}{*}{$\begin{array}{c}\text { All } \\
\text { grades }(\%\end{array}$} \\
\hline & 1 & 2 & 3 & 4 & \\
\hline \multicolumn{6}{|l|}{ Hematological } \\
\hline Leukopenia & 1 & 0 & 2 & 2 & $5(83.3)$ \\
\hline Neutropenia & 0 & 1 & 1 & 3 & $5(83.3)$ \\
\hline Thrombocytopenia & 2 & 0 & 1 & 1 & $4(66.7)$ \\
\hline Anemia & 1 & 2 & 1 & 0 & $4(66.7)$ \\
\hline \multicolumn{6}{|l|}{ Non-hematological } \\
\hline Increased creatinine & 1 & 0 & 0 & 0 & $1(16.7)$ \\
\hline Increased blood bilirubin & 2 & 1 & 0 & 0 & $3(50.0)$ \\
\hline Increased AST/ALT & 3 & 2 & 1 & 0 & $6(100.0)$ \\
\hline Constipation & 2 & 0 & 0 & 0 & $2(33.3)$ \\
\hline Diarrhea & 2 & 2 & 0 & 0 & $4(66.7)$ \\
\hline Fatigue & 2 & 0 & 1 & - & $3(50.0)$ \\
\hline Anorexia & 0 & 1 & 1 & 0 & $2(33.3)$ \\
\hline Nausea & 4 & 2 & 0 & - & $6(100.0)$ \\
\hline Vomiting & 1 & 0 & 0 & 0 & $1(16.7)$ \\
\hline Sensory neuropathy & 1 & 1 & 1 & 0 & $3(50.0)$ \\
\hline Alopecia & 1 & 0 & - & - & $1(16.7)$ \\
\hline
\end{tabular}

B, nab-PTX + GEM (n=18)

Grade, $\mathrm{n}$

Toxicities

\begin{tabular}{lccccc} 
Toxicities & 1 & 2 & 3 & 4 & grades $(\%)$ \\
\hline Hematological & & & & & \\
Leukopenia & 3 & 6 & 7 & 1 & $17(94.4)$ \\
Neutropenia & 1 & 3 & 6 & 4 & $14(77.8)$ \\
Thrombocytopenia & 8 & 4 & 1 & 0 & $13(72.2)$ \\
Anemia & 3 & 10 & 2 & 1 & $16(88.9)$ \\
Non-hematological & & & & & \\
Increased creatinine & 3 & 2 & 0 & 0 & $5(27.8)$ \\
Increased blood bilirubin & 3 & 1 & 1 & 0 & $5(27.8)$ \\
Increased AST/ALT & 7 & 3 & 3 & 0 & $13(76.5)$ \\
Constipation & 7 & 0 & 0 & 0 & $7(38.9)$ \\
Diarrhea & 4 & 1 & 0 & 0 & $5(27.8)$ \\
Fatigue & 7 & 1 & 0 & - & $8(44.4)$ \\
Dysgeusia & 4 & 0 & - & - & $4(22.2)$ \\
Anorexia & 1 & 3 & 0 & 0 & $4(22.2)$ \\
Stomatitis & 1 & 1 & 0 & 0 & $2(11.1)$ \\
Nausea & 2 & 2 & 0 & - & $4(22.2)$ \\
Vomiting & 2 & 0 & 0 & 0 & $2(11.1)$ \\
Skin rash & 4 & 1 & 0 & 0 & $5(27.8)$ \\
Edema & 1 & 0 & 0 & - & $1(5.6)$ \\
Sensory neuropathy & 5 & 3 & 1 & 0 & $9(50)$ \\
Hyperkalemia & 1 & 1 & 0 & 0 & $2(11.1)$ \\
Arthralgia & 3 & 0 & 0 & - & $3(16.7)$ \\
\hline & & & & &
\end{tabular}

Table IV. Continued.

C, GEM (n=11)

\begin{tabular}{|c|c|c|c|c|c|}
\hline \multirow[b]{2}{*}{ Toxicities } & \multicolumn{4}{|c|}{ Grade, $n$} & \multirow{2}{*}{$\begin{array}{c}\text { All } \\
\text { grades }(\%)\end{array}$} \\
\hline & 1 & 2 & 3 & 4 & \\
\hline \multicolumn{6}{|l|}{ Hematological } \\
\hline Leukopenia & 1 & 4 & 2 & 0 & $7(63.6)$ \\
\hline Neutropenia & 0 & 2 & 4 & 0 & $6(54.5)$ \\
\hline Thrombocytopenia & 2 & 3 & 0 & 0 & $5(45.6)$ \\
\hline Anemia & 1 & 5 & 4 & 0 & $10(90.9)$ \\
\hline \multicolumn{6}{|l|}{ Nonhematological } \\
\hline Increased creatinine & 2 & 2 & 0 & 0 & $4(36.4)$ \\
\hline Increased blood bilirubin & 3 & 1 & 0 & 0 & $4(36.4)$ \\
\hline Increased AST/ALT & 7 & 0 & 0 & 0 & $7(63.6)$ \\
\hline Constipation & 4 & 3 & 0 & 0 & $7(63.6)$ \\
\hline Diarrhea & 1 & 0 & 0 & 0 & $1(9.1)$ \\
\hline Fatigue & 3 & 0 & 0 & - & $3(27.3)$ \\
\hline Dysgeusia & 3 & 0 & - & - & $3(27.3)$ \\
\hline Anorexia & 0 & 1 & 1 & 0 & $2(18.2)$ \\
\hline Stomatitis & 1 & 0 & 0 & 0 & $1(9.1)$ \\
\hline Nausea & 1 & 1 & 0 & - & $2(18.2)$ \\
\hline Vomiting & 1 & 1 & 0 & 0 & $2(18.2)$ \\
\hline Edema & 3 & 0 & 0 & - & $3(27.3)$ \\
\hline
\end{tabular}

D, S-1 $(n=9)$

Grade, $\mathrm{n}$

All

Toxicities

$\begin{array}{llll}1 & 2 & 3 & 4\end{array} \quad \operatorname{grades}(\%)$

Hematological

Leukopenia

Thrombocytopenia

$\begin{array}{lllll}2 & 1 & 0 & 0 & 3(33.3)\end{array}$

Non-hematological

Increased AST/ALT

Diarrhea

Fatigue

Dysgeusia

Anorexia

Stomatitis

Nausea

Sensory neuropathy

Epiphora

$\begin{array}{lllll}2 & 0 & 0 & 0 & 2(22.2)\end{array}$

$\begin{array}{lllll}4 & 0 & 0 & 0 & 4(44.4)\end{array}$

$\begin{array}{lllll}4 & 0 & 0 & 0 & 4(44.4)\end{array}$

$420 \quad-6(66.7)$

20 - - $2(22.2)$

$\begin{array}{lllll}2 & 0 & 0 & 0 & 2(22.2)\end{array}$

$\begin{array}{lllll}2 & 0 & 0 & 0 & 2(22.2)\end{array}$

$\begin{array}{lllll}1 & 0 & 0 & - & 1(11.1)\end{array}$

$\begin{array}{lllll}3 & 0 & 0 & 0 & 3(33.3)\end{array}$

$200 \quad-\quad 2(22.2)$

FOLFIRINOX, 5-fluorouracil/leucovorin, oxaliplatin and irinotecan; nab-PTX, nabpaclitaxel; GEM, gemcitabine; S-1, tegafur/gimeracil/ oteracil potassium; AST, aspartate aminotransferase; ALT, alanine aminotransferase.

Cost-effectiveness analysis (per month). The cost-effectiveness ratio was $¥ 642,544.6$ /MST for the FOLFIRINOX, $¥ 470,790.5 / \mathrm{MST}$ for the nab-PTX + GEM, ¥81,832.0/MST for 
the GEM and $¥ 55,633.1 /$ MST for the S-1 regimen. The differences between the four regimens were found to be significant $(\mathrm{P}<0.0001$; Table III).

AE analysis. The major AEs are summarised in Table IV. For the nab-PTX + GEM regimen, these included leukopenia $(94.4 \%)$, anemia $(88.9 \%)$, neutropenia $(77.8 \%)$, aspartate transaminase/alanine transaminase (AST/ALT) increase $(76.5 \%)$ and thrombocytopenia (72.2\%). As regards neutropenia, $71.4 \%$ of the cases were grade $\geq 3$. For the GEM regimen, anemia (90.9\%), leukopenia (63.6\%), AST/ALT increase (63.6\%), constipation $(63.6 \%)$ and neutropenia $(54.5 \%)$ were the most common AEs. As regards neutropenia, $66.7 \%$ of the cases were grade $\geq 3$. For the $\mathrm{S}-1$ regimen, fatigue (66.7\%), AST/ALT increase $(44.4 \%)$ and diarrhea $(44.4 \%)$ were the most common AEs; however, none were grade $\geq 3$.

\section{Discussion}

An analysis was conducted to compare cost-effectiveness among the FOLFIRINOX, nab-PTX + GEM, GEM and S-1 regimens for the treatment of metastatic pancreatic cancer. The nab-PTX + GEM regimen was considered to be the treatment with the highest therapeutic effectiveness and exhibited a similar effectiveness to the FOLFIRINOX regimen. However, in terms of cost-effectiveness, the nab-PTX + GEM regimen was superior to the FOLFIRINOX regimen. Previous studies by Gharaibeh et al (12) and Zhou et al (13) reported similar findings. However, it was reported that the FOLFIRINOX regimen was superior in terms of progression-free survival (11). Conversely, the GEM regimen exhibited inferior efficacy compared with the nab-PTX + GEM and FOLFIRINOX regimens $(2,4)$. In the present study, the MST of the S-1 regimen was found to be superior to the that of the GEM regimen, which was consistent with previous reports. There is evidence the efficacy of the S-1 regimen was non-inferior to that of the GEM regimen (3). However, the cost-effectiveness of the GEM and S-1 regimens were equivalent, and both were superior to the nab-PTX + GEM and FOLFIRINOX regimens. Zhou et al (13) reported that the S-1 regimen exhibited an excellent cost-effectiveness in the Gemcitabine and the TS-1 Trial (GEST). Similarly, Kurihara et al (11) reported that the S-1 regimen was superior in terms of cost-effectiveness.

Since the present study did not take patients' quality of life (QOL) into consideration, it is impossible to accurately determine cost-effectiveness. However, upon examining AEs, a high incidence of reduced QOL was hypothesized, particularly among patients who received the FOLFIRINOX and nab-PTX + GEM regimens. The FOLFIRINOX regimen may be particularly toxic and the AEs may be more severe compared with those observed with standard therapy using nab-paclitaxel plus gemcitabine, gemcitabine and S-1 regimens. The FOLFIRINOX regimen is associated with severe AEs, such as myelosuppression, nausea, fatigue and peripheral neuropathy. Okusaka et al (17), however, reported that the FOLFIRINOX regimen may be considered as a standard regimen and exhibited an acceptable toxicity profile in chemotherapy-naïve Japanese patients with metastatic pancreatic cancer. The FOLFIRINOX regimen has a high cost associated with supportive care, whereas the cost of the GEM and S-1 regimens for supportive care was the lowest among the regimens. Therefore, the use of the FOLFIRINOX regimen may become more limited in the future. Furuse et al (18) advocated for the adaptation of the FOLFIRINOX regimen over the nab-PTX + GEM regimen for non-elderly patients and patients with a good overall prognosis.

In conclusion, to the best of our knowledge, this is the first study in which the cost-effectiveness of four types of chemotherapy regimens for metastatic pancreatic cancer chemotherapy was analyzed. The nab-PTX + GEM and FOLFIRINOX regimens were associated with a high efficacy and high cost. By contrast, the GEM regimen exhibited a lower efficacy compared with the nab-PTX + GEM and FOLFIRINOX regimens, but the findings of this study suggest that the GEM and S-1 were the most cost-efficient regimens.

\section{References}

1. Von Hoff DD, Ervin T, Arena FP, Chiorean EG, Infante J, Moore M, Seay T, Tjulandin SA, Ma WW, Saleh MN, et al: Increased survival in pancreatic cancer with nab-paclitaxel plus gemcitabine. N Engl J Med 369: 1691-1703, 2013.

2. Ueno H, Ioka T, Ikeda M, Ohkawa S, Yanagimoto H, Boku N, Fukutomi A, Sugimori K, Baba H, Yamao K, et al: Randomized phase III study of gemcitabine plus S-1, S-1 alone, or gemcitabine alone in patients with locally advanced and metastatic pancreatic cancer in Japan and Taiwan: GEST study. J Clin Oncol 31: 1640-1648, 2013

3. Conroy T, Desseigne F, Ychou M, Bouché O, Guimbaud R, Bécouarn Y, Adenis A, Raoul JL, Gourgou-Bourgade S, de la Fouchardière $\mathrm{C}$, et al: FOLFIRINOX versus gemcitabine for metastatic pancreatic cancer. N Engl J Med 364: 1817-1825, 2011.

4. Von Hoff DD, Ramanathan RK, Borad MJ, Laheru DA, Smith LS, Wood TE, Korn RL, Desai N, Trieu V, Iglesias JL, et al: Gemcitabine plus nab-paclitaxel is an active regimen in patients with advanced pancreatic cancer: A phase I/II trial. J Clin Oncol 29: 4548-4554, 2011.

5. Yamaguchi K, Okusaka T, Shimizu K, Furuse J, Ito Y, Hanada K, Shimosegawa T, Okazaki K; Committee for Revision of Clinical Guidelines for Pancreatic Cancer of the Japan Pancreas Society: Clinical Practice Guidelines for Pancreatic Cancer 2016 From the Japan Pancreas Society: A Synopsis. Pancreas 46: 595-604, 2017.

6. Saltz LB, Clarke S, Díaz-Rubio E, Scheithauer W, Figer A, Wong R, Koski S, Lichinitser M, Yang TS, Rivera F, et al: Bevacizumab in combination with oxaliplatin-based chemotherapy as first-line therapy in metastatic colorectal cancer: A randomized phase III study. J Clin Oncol 26: 2013-2019, 2008.

7. Van Cutsem E, Köhne CH, Láng I, Folprecht G, Nowacki MP, Cascinu S, Shchepotin I, Maurel J, Cunningham D, Tejpar S, et al: Cetuximab plus irinotecan, fluorouracil, and leucovorin as first-line treatment for metastatic colorectal cancer: Updated analysis of overall survival according to tumor KRAS and BRAF mutation status. J Clin Oncol 29: 2011-2019, 2011.

8. Bokemeyer C, Bondarenko I, Hartmann JT, de Braud F, Schuch G, Zubel A, Celik I, Schlichting $M$ and Koralewski P: Efficacy according to biomarker status of cetuximab plus FOLFOX-4 as first-line treatment for metastatic colorectal cancer: The OPUS study. Ann Oncol 22: 1535-1546, 2011.

9. Meropol NJ, Schrag D, Smith TJ, Mulvey TM, Langdon RM Jr, Blum D, Ubel PA and Schnipper LE, American Society of Clinical Oncology: American Society of Clinical Oncology guidance statement: The cost of cancer care. J Clin Oncol 27: 3868-3874, 2009.

10. Carrato A, García P, López R, Macarulla T, Rivera F, Sastre J, Gostkorzewicz J, Benedit P and Pérez-Alcántara F: Cost-utility analysis of nanoparticle albumin-bound paclitaxel (nab-paclitaxel) in combination with gemcitabine in metastatic pancreatic cancer in Spain: Results of the PANCOSTABRAX study. Expert Rev Pharmacoecon Outcomes Res 15: 579-589, 2015.

11. Kurihara T, Kobayashi M, Kogo M, Yoneyama K, Ito N, Sunaga T, Konishi K, Imawari M, Tobe T and Kiuchi Y: Cost-effectiveness analysis of chemotherapy with GEM or S-1 for patients with non-resectable pancreatic cancer. Gan To Kagaku Ryoho 37: 659-664, 2010. (In Japanese) 
12. Gharaibeh M, Bootman JL, McBride A, Martin J and Abraham I: Economic evaluations of first-Line chemotherapy regimens for pancreatic cancer: A critical review. Pharmacoeconomics 35: 83-95, 2016.

13. Zhou J, Zhao R, Wen F, Zhang P, Tang R, Du Z, He X, Zhang J and Li Q: Cost-effectiveness analysis of gemcitabine, S-1 and gemcitabine plus $\mathrm{S}-1$ for treatment of advanced pancreatic cancer based on GEST study. Med Oncol 32: 121, 2015.

14. Zhou J, Zhao R, Wen F, Zhang P, Wu Y, Tang R, Chen H, Zhang J and Li Q: Cost-effectiveness analysis of treatments for metastatic pancreatic cancer based on PRODIGE and MPACT trials. Tumori 3: 294-300, 2016.

15. Kawakami Y: Medical fee points table. 35 th edition. Social Insurance Institute, Tokyo, 2008
16. Pharmaceutical Society: Insurance drug encyclopedia. Pharmaceutical Society, Tokyo, 2012.

17. Okusaka T, Ikeda M, Fukutomi A, Ioka T, Furuse J, Ohkawa S, Isayama $\mathrm{H}$ and Boku N: Phase II study of FOLFIRINOX for chemotherapy-naïve Japanese patients with metastatic pancreatic cancer. Cancer Sci 105: 1321-1326, 2014.

18. Furuse J: Up to date of chemotherapy for pancreatic cancer.Nihon Shokakibyo Gakkai Zasshi 114: 637-643, 2017 (In Japanese). 